\title{
Computed Tomographic Evaluation of Posterior Fossa Lesions
}

\author{
Gupta MK ${ }^{1}$, Rauniyar RK ${ }^{1}$, Bhatta $N^{2}$, Raja $S^{3}$, Ahmad $K^{1}$ \\ ${ }^{1}$ Department of Radiodiagnosis \& Imaging, ${ }^{2}$ Department of Internal Medicine, ${ }^{3}$ Department \\ of Pediatrics, B.P. Koirala Institute of Health Sciences, Dharan, Nepal.
}

\begin{abstract}
Background: Various neoplastic and nonneoplastic lesions can involve posterior cranial fossa. Posterior fossa lesions are potentially fatal since they may result in compression of the brainstem. The objective of this study was to analyze the spectrum of posterior fossa lesions and their clinical presentations. Methods: A prospective cross sectional study was conducted over a period of one year. Thirty patients with clinical features of posterior fossa pathology, referred for cranial computed tomography, were evaluated in the study. Results: Posterior fossa lesions found in this study were cerebellar abscess- 4(13.3\%), arachnoid cyst$4(13.3 \%)$, brainstem glioma- 3(10\%), cerebellar astrocytoma- 3(10\%), acute cerebellar infarct- 2(6.7\%), cerebellar metastasis- 2(6.7\%), acoustic neuroma- 2(6.7\%), meningioma2(6.7\%), medulloblastoma- 2(6.7\%), ependymoma- 1(3.3\%), Dandy-Walker malformation$1(3.3 \%), 4^{\text {th }}$ ventricular bleed- $1(3.3 \%)$, brainstem haemorrhage- $1(3.3 \%)$, cerebellar haemorrhage- $1(3.3 \%)$, and neurocysticercosis- 1(3.3\%). The maximum numbers of cases $(23.3 \%)$ with posterior fossa lesions were in their first decade of life. Features of raised intracranial pressure (i.e. headache, nausea \& vomiting) were the commonest presenting symptoms. Other symptoms included altered sensorium, ataxia, fever, limb weakness, vertigo, seizure, hearing loss, neck stiffness, slurring of voice, ear discharge, tinnitus, loss of consciousness and macrocrania in decreasing order of frequency. Conclusion: There is wide spectrum of the lesions in posterior fossa in children and adults in this part of Nepal, requiring prompt diagnosis and intervention, and $\mathrm{CT}$ has a pivotal role in the management.
\end{abstract}

Keywords: Computed Tomography, Posterior Fossa Lesions, Tumors

\section{Introduction}

The posterior cranial fossa contains cerebellum and brain stem which is outlined

Correspondence to: Dr. Mukesh Kumar Gupta, Associate Professor, Department of Radiodiagnosis and Imaging, BPKIHS, Dharan, Nepal

E-mail:- mukeshgupta148@yahoo.com by the clivus, petrous and occipital bones. Various neoplastic and nonneoplastic lesions can involve the posterior fossa. These are further divided into extraaxial (acoustic Neuroma, meningioma, epidermoid tumours, arachnoid cyst, Dandy-Walker cyst, DandyWalker variant, chordoma, metastases, vascular abnormalities, infective lesions, traumatic extraaxial collections) and intraaxial lesions (brainstem gliomas, 
cerebellar astrocytoma, medulloblastoma, ependymoma, choroid plexus pappilloma, hemangioblastoma, metastases, hemorrhage, infarct, multiple sclerosis, infective lesions, traumatic intraaxial lesions). ${ }^{1}$ Though MRI is the imaging modality of choice for evaluation of the posterior fossa, because of the low cost and universal availability, CT is still initial investigation of choice at many centers. Calcification within a mass often cannot be appreciated on the MR scan. In these cases, CT scan, with its extraordinary sensitivity to detect even small amount of calcification, has been more helpful than $\mathrm{MR} .^{2}$ Bone-window settings on CT can also reveal bone erosion or hyperostosis, associated with extra-axial tumors. The purpose of this study was to analyze the spectrum of posterior fossa lesions and their clinical presentations.

\section{Methods}

A prospective cross sectional study was conducted on 30 patients in the department of Radiodiagnosis \& Imaging, B. P. Koirala Institute of Health Sciences, Dharan, Nepal over a period of one year. All the patients with clinical features of posterior fossa lesions referred for CT head were included in the study. After taking informed consent, detail history was taken and clinical examination was done in each patient. Noncontrast CT (NCCT) and contrast enhanced CT (CECT) scan of the head was done on SIEMENS SOMATOM AR STAR taking $3 \times 3 \mathrm{~mm}$ axial sections at the base and 5 x 5 axial sections above. Intravenous contrast was avoided in patient with history of drug allergy \& renal dysfunction. Diagnosis was made on the basis of characteristic CT features of the lesions and post-operative biopsy/response to the treatment during follow-up visits. Statistical analysis was performed using SPPS software.

\section{Results}

Of the total 30 patients in our study, $19(63.3 \%)$ were adult and 11(36.7\%) were children, and the age ranged from 1 to 76 years. The maximum numbers of cases $(23.3 \%)$ with posterior fossa lesions were in their first decade of life. The spectrum of posterior fossa lesions on CT scan were as follows: Cerebellar abscess- 4(13.3\%), arachnoid cyst- 4(13.3\%), Brainstem glioma$3(10 \%)$, cerebellar astrocytoma- 3(10\%), acute cerebellar infarct- 2(6.7\%), cerebellar metastasis- 2(6.7\%), acoustic neuroma2(6.7\%), meningioma- 2(6.7\%), medulloblastoma- 2(6.7\%), ependymoma1(3.3\%), Dandy-Walker malformation$1(3.3 \%), 4^{\text {th }}$ ventricular bleed- $1(3.3 \%)$, brainstem haemorrhage- 1(3.3\%), cerebellar haemorrhage- $1(3.3 \%)$, neurocysticercosis$1(3.3 \%)$.

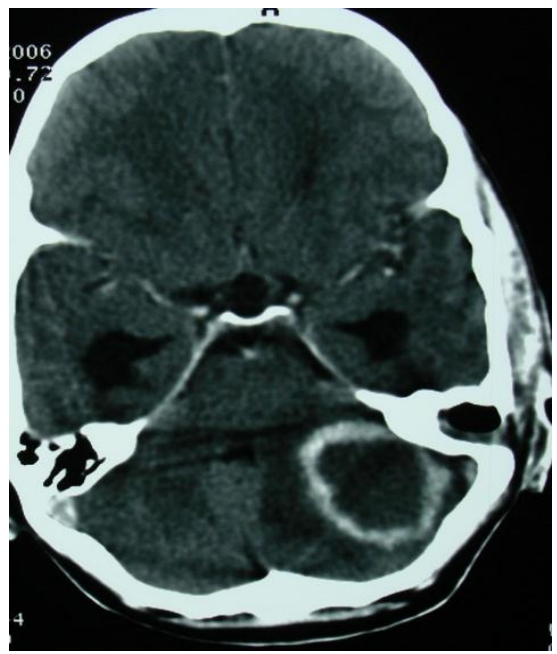

Fig 1: Left cerebellar abscess. Axial CECT showing thick walled peripherally enhancing hypodense lesion with surrounding edema in left cerebellar hemisphere. 
Spectrum of posterior fossa lesions in children: Cerebellar abscess- 3(27.2\%), cerebellar astrocytoma- 2(18.1\%), brainstem glioma- 2(18.1\%), medulloblastoma$2(18.1 \%)$, ependymoma- 1(9\%), DandyWalker Malformation- 1(9\%).

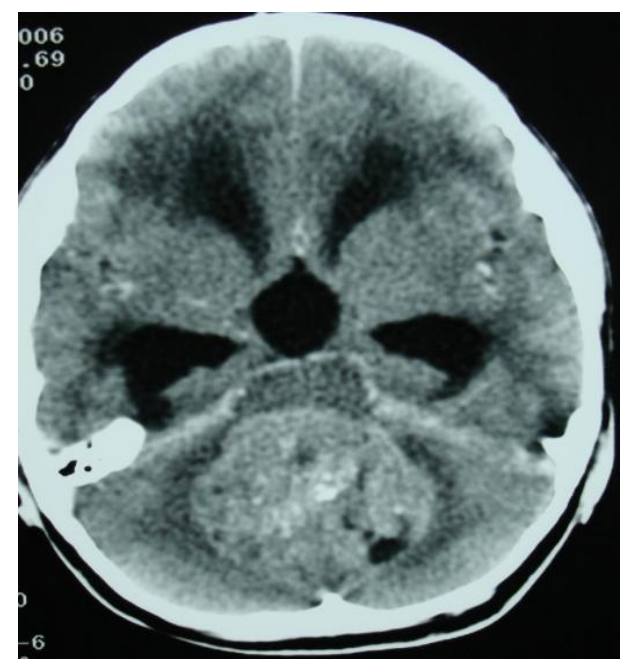

Fig 2: Medulloblastoma. CT scan shows a well-defined inhomogeneous enhancing hyperdense lesion with foci of calcification in cerebellar vermis filling the $4^{\text {th }}$ ventricle and causing obstructive hydrocephalus.

Spectrum of posterior fossa lesions in adult: Arachnoid cyst- 4(21.0\%), acute cerebellar infarct- 2(10.5\%), cerebellar metastasis$2(10.5 \%)$, acoustic neuroma- 2(10.5\%), meningioma- $2(10.5 \%)$, brainstem glioma$1(5.2 \%)$, cerebellar abscess- 1(5.2\%), cerebellar astrocytoma- 1(5.2\%), 4th ventricular bleed- 1(5.2\%), brainstem haemorrhage- $\quad 1(5.2 \%)$, cerebellar haemorrhage- $1(5.2 \%)$, neurocysticercosis$1(5.2 \%)$.

The clinical findings in patient with posterior fossa lesions were as follows: Headache21(70.0\%), nausea \& vomiting- 17(56.7\%), altered sensorium- 13(43.3\%), ataxia$9(30 \%)$, fever- $5(16.7 \%)$, limb weakness$5(16.7 \%)$, vertigo- $4(13.3 \%)$, seizure$4(13.3 \%)$, hearing loss- $4(13.3 \%)$, neck stiffness- $2(6.7 \%)$, slurring of voice$2(6.7 \%)$, ear discharge- $2(6.7 \%)$, tinnitus2(6.7\%), macrocrania- $1(3.3 \%)$. The commonest site of posterior fossa lesions was cerebellum (40\%) in both children and adults. The cerebellum was involved in $4(36.3 \%)$ cases out of total 11 cases in children and in $8(42.1 \%)$ cases out of total 19 cases in adults.

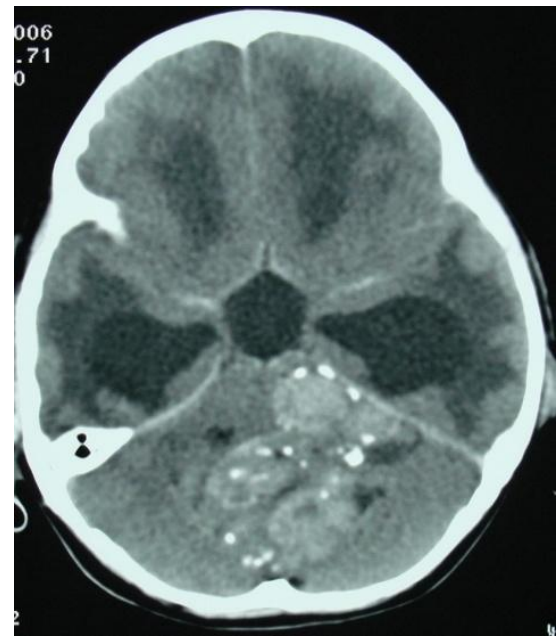

Fig 3: Ependymoma. CT scan shows a hyperdense lesion with punctate calcification filling the $4^{\text {th }}$ ventricle and extending into left $\mathrm{CP}$ angle cistern with associated obstructive hydrocephalus.

\section{Discussion}

Posterior cranial fossa lesions are considered critical brain lesions because of the limited space and potential involvement of brain stem. About one third of posterior fossa neoplasms in children are cerebellar astrocytoma, one third to one quarter are PNET/medulloblastoma, one fourth are brainstem glioma, and one eighth are ependymomas. ${ }^{2,3}$ The three most common primary posterior fossa neoplasms in adults are extraaxial i.e. schwannoma, meningioma, and epidermoid tumours. Metastasis is the commonest intraaxial posterior fossa tumor in adults. Haemangioblastoma and 
brainstem gliomas are two most common primary intraaxial neoplasms in adults. ${ }^{4}$

Reider-Groswasser et $\mathrm{al}^{5}$ found posterior fossa abnormalities in 190 of 7000 brain CT studies. Of these, $51.5 \%$ were primary tumors, $15.3 \%$ metastasis, $12.1 \%$ vascular lesions and $5.8 \%$ congenital or developmental lesions. In our study, $43.3 \%$ were primary tumors, $16.7 \%$ were cyst \& cyst like malformations i.e. congenital or developmental lesions, $13.3 \%$ were cerebellar abscess, $10 \%$ were non traumatic bleed, $6.7 \%$ were metastasis, $6.7 \%$ were cerebellar infarct, and $3.3 \%$ were neurocysticercosis.

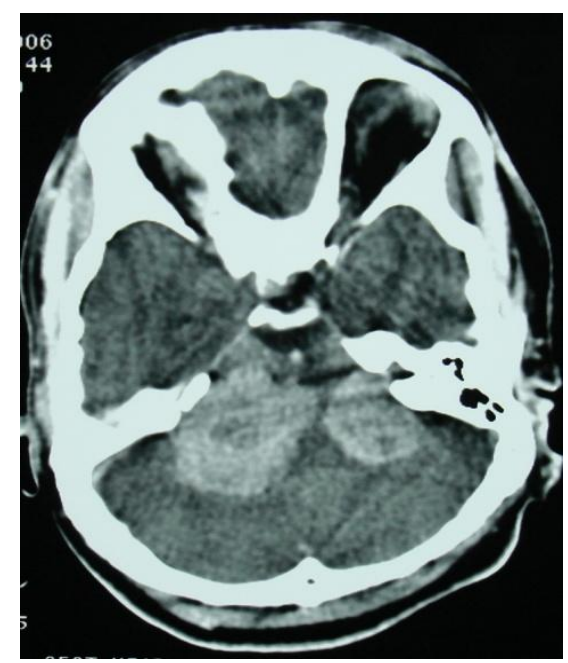

Fig 4: Bilateral Acoustic Neuroma. CT scan shows intensely enhancing mass with small intratumoral cystic component in bilateral $\mathrm{CP}$ angles.

Seven $\mathrm{H}_{\text {et }} \mathrm{al}^{6}$ reported intracranial abscesses in 32 cases of 780 patients of chronic suppurative otitis media (CSOM). Perisinus abscess (13 of 32) was the commonest abscess, followed by temporal lobe abscess (8 of 32), epidural abscess (7 of 32), cerebellar abscess (6 of 32) and subdural empyema (2 of 32). Headache (93\%), fever $(87 \%)$ and altered mental status $(62 \%)$ were the most common presenting features, along with symptoms of CSOM.

In our study there were 4 cases of cerebellar abscess (fig. 1) of which $3(75 \%)$ were in children and $1(25 \%)$ in adult. Headache, nausea, vomiting, fever, altered sensorium, hearing loss, neck rigidity, ear discharge, vertigo \& seizure were the common presentations in decreasing order of frequency. Among these 4 cases, 2(50\%) were secondary to CSOM and rest $2(50 \%)$ were due to the complication of meningoencephalitis.

Badhe et $\mathrm{al}^{7}$ observed $51 \%$ of brain stem gliomas occurring in first decade of life in a study consisting of 45 cases of brain stem glioma. The female to male ratio was 1.04:1. The commonest presenting features were cranial nerve palsies (33\%) and cerebellar signs $(29.8 \%) .55 .55 \%$ of cases were located in the pons, $31.01 \%$ in the medulla and $13.33 \%$ in the midbrain.

There were 3 cases of brainstem glioma in our study with child to adult ratio of 2:1. Ataxia (100\%) was the commonest presenting feature followed by headache (66.6\%), nausea \& vomiting (33.3\%), weakness of the limb (33.3\%), and slurring of the voice $(33.3 \%)$. Both medulla and pons were involved in $66.6 \%$ and only medulla in $33.3 \%$ of the cases of brain stem glioma.

Medulloblastoma is the most common malignant pediatric brain tumor, accounting for 15 to $20 \%$ of pediatric brain tumors and 30 to $55 \%$ of posterior fossa tumors. Mean age at presentation is 5 to 7 years, and $80 \%$ of patients are younger than $15 .{ }^{8,9,10}$ There were 2 cases of medulloblastoma in our study and the both presented in first decade 
of life. In our study, medulloblastoma (fig. 2) accounted for $28.5 \%$ of the posterior fossa tumors in children. Features of raised intracranial pressure (i.e. headache, nausea $\&$ vomiting), seizure and ataxia were the common clinical presentations. Cerebellar astrocytoma accounts for more than $10 \%$ of pediatric intracranial tumors and $25 \%$ of all posterior fossa tumors in children. It usually manifests as signs of raised intracranial pressure and/ or obstructive hydrocephalus and occasionally with cerebellar signs such as ataxia. The mean age of presentation for cerebellar astrocytoma is 8 to 9 years. ${ }^{11,12,13}$

In our study there were 3 cases of cerebellar astrocytomas with child to adult ratio of 2:1. Cerebellar astrocytomas accounted for $28.5 \%$ of total posterior fossa tumors in children and $16.6 \%$ of total posterior fossa primary tumors in adults. The common presentations were headache $(100 \%)$, nausea $\&$ vomiting $(100 \%)$, ataxia (100\%), slurring of voice $(33.3 \%)$ and altered sensorium $(33.3 \%)$. Obstructive hydrocephalus was noted in all of the cases.

Ependymomas constitute $15 \%$ of posterior fossa neoplasm in childhood and are the third most common pediatric brain tumor. They are four to six times more common in children as compared to adults. The peak age range is 1 to 5 years but there is a second, smaller peak in the mid-thirties. Common presenting features are disequilibrium, nausea, vomiting, headache, ataxia and nystagmus. ${ }^{14,15}$

The commonest neoplasm in the cerebellum in adults is metastasis. Thirty percent of all CNS tumors are metastases and $20 \%$ of all metastases occur in the posterior cranial fossa. ${ }^{16,17,18}$
Tumors of the posterior fossa are more common in children than adults. About 54 to $70 \%$ of all childhood brain tumors originate in the posterior fossa as compared with 15 to $20 \%$ in adults The clinical presentations of posterior fossa tumors result by two mechanisms: the first mechanism is obstruction of the normal cerebrospinal fluid (CSF) pathway, while direct infiltration and/or mass effect on the surrounding structures (cerebellum, cranial nerves or their nuclei and long tracts) represents the second mechanism responsible for manifestations of posterior fossa tumors. ${ }^{19,20}$

\section{Conclusion}

There is wide spectrum of the lesions in posterior cranial fossa in children and adults, requiring prompt diagnosis and intervention and CT has a pivotal role in the management. The vast majority of posterior fossa tumors are extraaxial in adults. Conversely, in childhood, the majority of posterior fossa masses are intraaxial or intraventricular.

\section{References}

1. Ramsey RG. Neuroradiology. Third Edition. 1994:495-564.

2. Harwood-Nash DC. Primary neoplasms of the central nervous system in children. Cancer 1991;67:1223-1228.

3. Gusnard DA. Cerebellar neoplasm in children. Sem Roentgenol 1990;25:263278.

4. Lizak PF, Woodruff WW. Posterior fossa neoplasm: Multiplanar imaging. Semin US, CT, MR 1992;13:182-206. 
5. Reider-Groswasser I, Catz A, Luz I. Computerized tomography findings in posterior fossa lesions. Comput Radiol 1986;10:311-8.

6. Seven H, Coskun BU, Calis AB, Sayin I, Turgut S. Intracranial abscesses associated with chronic suppurative otitis media. Eur Arch Otorhinolaryngol 2005;262:847-51.

7. Badhe PB, Chauhan PP, Mehata NK. Brainstem gliomas--a clinicopathological study of 45 cases with p53 immunohistochemistry. Indian $\mathrm{J}$ Cancer 2004;41:170-4.

8. Maher CO, Friedman JA, Raffel C. Posterior Fossa Tumors in Children. In: Batjer HH, Loftus CM (eds). Textook of Neurological Surgery: Principles and Practice. Philadelphia: Lippincott Williams \& Wilkins; 2003. p. 985.

9. Roberts RO, Lynch CF, Jones MP, Hart MN. Medulloblastoma: A populationbased study of 532 cases. J Neuropathol Exp Neurol 1991;50:134-144.

10. Rutka JT, Kuo JS. Pediatric surgical neuro-oncology: Current best care practices and strategies. J Neurooncol 2004;69:139-150.

11. Zimmerman R, Bilaniuk LT, Bruno L. Computed tomography of cerebellar astrocytoma. Am J Roentgenol 1978;130:929-33.

12. Tew JM, Feibel JH, Sawaya R. Brain tumors: Clinical aspects. Semin Roentgenol 1984;19:115-128.
13. Farwell JR, Dohrmann GJ, Flannery JT. Central nervous system tumors in children. Cancer 1977;40:3123-3132.

14. Nazar GB, Hoffman HJ, Becker LE, Jenkin D, Humphreys RP, Hendrick EB. Infratentorial ependymomas in childhood: Prognostic factors and treatment. J Neurosurg 1990;72:408-417.

15. Lyons MK, Kelly PJ. Posterior fossa ependymoma: Report of 30 cases and review of the literature. Neurosurg 1991;28:659-672.

16. Gado M, Huete I, Mikhael M. Computerised tomography of infratentorial tumors. Semin Roentgenol 1977;12:109-120.

17. Baker HLJ, Houser OW, Campbell JK. National cancer Institute study: Evaluation of computed tomography in the diagnosis of intracranial neoplasms. Radiology 1980;136:91-96.

18. Weisberg LA. Solitary cerebellar metastasis: Clinical and computed tomographic correlations. Arch Neurol 1985;42:336-341.

19. Bidiwala S, Pittman T. Neural Network Classification of Pediatric Posterior Fossa Tumors. Pediatr Neurosurg 2004;40:8-15.

20. Sgouros S, Fineron PW, Hockley AD. Cerebellar astrocytomas of the childhood: Long term follow up. Child's Nerv Syst 1995;11:89-96. 